\title{
Bibliometría: un insumo para la escritura científica en Ingeniería
}

\section{Bibliometry: an input for the scientific writing in Engineering}

\author{
Carlos Alberto Montero Corrales \\ Universidad de Costa Rica, Escuela de Administración Pública; San José, Costa Rica. \\ carlos.monterocorrales@gmail.com \\ https://orcid.org/0000-0001-6368-6044
}

Referencia/ reference:

Montero, C. (2020). Bibliometría: un insumo para la escritura científica en Ingeniería. Yulök Revista de Innovación Académica, 4(2). 98-103. https://doi.org/10.47633/yulk.v4i2.237

Recibido: 9 de mayo del 2020

Aceptado:1 de diciembre del 2020

\section{Resumen}

El artículo de investigación tiene el objetivo de analizar las líneas en el campo de la ingeniería establecidas en el Plan Estratégico 2019-2025 del Instituto de Investigaciones en Ingeniería (INII) de la Universidad de Costa Rica (UCR), por medio de la aplicación de modelos matemáticos y estadísticos a la producción científica indexada en Elsevier Scopus, con el fin de orientar estratégicamente la producción científica. El artículo evidencia un estudio de caso acerca de la innovación tecnológica del proceso para la indagación de la producción científica mundial de artículos científicos y congresos en áreas específicas de la ingeniería. La metodología se desarrolló en cuatro etapas: 1. Aplicación de una entrevista semiestructurada, 2. Estudio de los resultados científicos en la base de datos Elsevier Scopus, 3. Visualización estadística de los resultados y 4 . Análisis de los resultados bibliométricos. Los resultados del estudio muestran quienes son las personas investigadoras que más publican en las redes de conocimiento mundiales acerca de creación de un sistema versátil para entender y controlar objetos, control de proceso, validación con equipo experimental, materiales de referencia, valoración de impacto en tiempo real, infraestructura sostenible, medición de oleaje y aprovechamiento de recursos renovables. Una conclusión principal es que el estudio bibliométrico en el INII contribuyó a orientar eficientemente las acciones para analizar, producir y difundir artículos científicos y participar en congresos internacionales.

Palabras clave: Bibliometría, Evaluación, Innovación de Proceso, Resultado de Búsqueda, Planificación.

\section{Abstract}

The paper aims to analyze the research lines in the field of engineering established in the 2019-2025 Strategic Plan of the Engineering Research Institute (INII, by its acronym in Spanish) of the University of Costa Rica (UCR by its acronym in Spanish), through the application of mathematical and statistical models to the indexed scientific production in Elsevier Scopus, in order to strategically guide scientific production. The article evidences a case study about the technological innovation of the process for the research of world scientific production of papers and congresses in specific areas of the engineering. The methodology was developed in four stages: 1. Application of a semi-structured interview, 2. Study of the scientific results in the Elsevier Scopus database, 3. Statistical visualization of results, and 4. Bibliometric analyses of the results. The results of the study show who are the researchers who publish the most in world knowledge networks about: creation of a versatile systems to understand and control objects, process control, validation with experimental equipment, reference materials, impact assessment on real time, sustainable infrastructure, wave measurement and use of renewable resources. A main conclusion is that the bibliometric study at the INII contributed to efficiently orient the actions to analyze, produce and disseminate scientific articles and participate in international congresses.

Keywords: Bibliometry, Evaluation, Process Innovation, Research Results, Planning. 


\section{Introducción}

El Instituto de Investigaciones en Ingeniería (INII) tiene treinta y nueve años de existencia y durante este tiempo se han realizado esfuerzos para planificar estratégicamente las áreas de su quehacer (Blanco, 2004), sin embargo, en la actualidad se hizo necesario transformar estos esfuerzos de planificación en una estrategia coherente para que los laboratorios puedan producir ingeniería que responda a los nuevos desafíos que enfrenta la sociedad costarricense y global.

Ante esta necesidad, a finales del año 2018 el INII inició el desarrollo del Plan Estratégico Institucional 20192025, por medio del cual se fortalecieron, entre otras, las capacidades para generar conocimiento por medio de la publicación de artículos científicos (V. Schmidt, comunicación personal, 5 de diciembre 2018). Como parte de las acciones para desarrollar dicho plan, se realizó esta investigación que tuvo como problema: ¿de qué manera se puede orientar la actividad científica del Instituto, con el fin de que la escritura de artículos y la participación en congresos sea de mayor impacto en el área del conocimiento ingenieril? Esta pregunta se respondió con la hipótesis: el estudio de las publicaciones científicas indexadas en Elsevier Scopus es una herramienta eficaz para orientar la escritura de artículos científicos y la participación en congresos en el área del conocimiento de la ingeniería. El objetivo de la investigación fue analizar las líneas de investigación prioritarias del INII, por medio de la aplicación de modelos matemáticos en la producción científica indexada en Elsevier Scopus, con el fin de orientar estratégicamente la investigación. Para esto se realizaron cuatro etapas, las cuales se explicarán a continuación.

\section{Metodología}

La metodología parte del aporte teórico de Price (1963) sobre la categoría ciencia, entendida como aquello que se edita en las publicaciones científicas, lo que permite medir la actividad de la ciencia tomando en consideración las características de las publicaciones, lo cual según Price (1963) era la forma para conectar la investigación con la comunidad científica. A partir de este concepto de ciencia, se desprende la bibliometría, que se basa en el cálculo y en el análisis de los resultados de búsqueda de lo que es cuantificable en la producción y en el consumo de la información científica (López, 1972; Spinak, 1996); la cual ayuda a explorar, organizar y analizar grandes cantidades de datos e identifica "patrones ocultos" que les permiten a las personas investigadoras tomar decisiones acerca de la escritura científica (Daim et al., 2006). Con la finalidad de realizar una metodología acorde con estas premisas teórico-metodológicas, a continuación, se explicarán las cuatro etapas de la investigación. Así mismo, el método subsecuente se presenta como una innovación tecnológica de proceso, ya que se introdujo una nueva tecnología para el procesamiento de información científica destinada a mejorar la calidad de la orientación estratégica de la escritura en el campo de conocimiento de las ingenierías en el INII.

Por lo tanto, la primera etapa se basó en la aplicación de una entrevista semiestructurada a los siete equipos de investigación, uno por cada laboratorio del INII, en donde se priorizó una línea de investigación en cada laboratorio para realizar el estudio bibliométrico. Con las palabras claves generadas en la entrevista, se construyeron ecuaciones de búsqueda que se ejecutaron en la base de datos de Elsevier Scopus, tomando en consideración operadores booleanos de búsqueda expresados por "AND", "OR" y “AND NOT" (Villegas, 2003). El operador "AND” funcionó para encontrar resultados de búsqueda en los que ambas palabras se encontraron al mismo tiempo, ya sea en el título, en el resumen o en las palabras claves de los resultados de búsqueda. El "OR" funcionó para encontrar una palabra u otra, sin generar ningún condicionante. Finalmente, el operador "AND NOT" para excluir palabras de los resultados de búsqueda. También, se utilizaron las comillas " " para buscar frases completas en la ecuación. Según lo anterior, se construyeron y validaron las siguientes ecuaciones:

1. ARCOS-Lab: "Robotic" AND "humanoid robot" AND ("object model" AND "manipulation") OR "cognitive architecture" OR "Impedance Control" OR "Cognitive Manipulation" OR "Complex Task" OR "Affordance" OR "ABSMachine Learning" OR "KEYMachine Learning") AND NOT "Imitation" AND NOT "Teleoperation" AND NOT "Developmental Robot".

2. CERLAB: "Process Control" AND "PID Control" AND "Optimization" OR "Modelling" OR "Model Predictive Control" OR "System Identification" AND NOT "Control Theory" AND NOT "Social Sciences".

3. LABCAL: "Metrology" AND "Reference Material" AND "Standard" OR "Calibration" OR "Verification" OR "Legal Metrology" AND NOT "Medical" AND NOT "Laws" AND NOT "Marketplace" AND NOT ALL "Biology" AND NOT ALL "Astronomy". 
4. LIS: "earthquake" AND "damage" AND "Accelerometer" OR "Near Real Time" OR "Strong Motion Network" OR "loss assessment" OR "intensity measures" AND NOT "Multi Hazard" AND NOT "Landslides" AND NOT "Tsunami".

5. Hidráulica: "Stormwater" AND "Low Impact Development" AND NOT "Reservoir" AND NOT "Architecture" AND NOT "Ecology" AND NOT "Biodiversity".

6. IMARES: "Water Waves" AND "Waves Measurement" OR "Physical Modelling" OR "Climate Waves" OR "Waves Gauges" OR " Waves Analyses" AND NOT "Animals" AND NOT "Biology".

7. REFORESTA: "Biological Materials" AND "Biological Products" OR "Energy" OR "Biomass" OR "Biofuels" OR "Bioindicator" AND NOT "Fossil Fuels" AND NOT "Hydraulic Energy" AND NOT "Solar Energy" AND NOT "Biodigestion" AND NOT "Public Policy".

Estas ecuaciones de búsqueda se ejecutaron en Elsevier Scopus, lo cual generó una base de datos con resultados de búsqueda correspondientes a cada línea de investigación. Asimismo, a estas bases de datos se les realizaron algunas preguntas, las cuales generaron los resultados que se mostrarán en la siguiente sección. La tercera etapa consistió en la visualización estadística de los resultados, en donde se tomó en consideración los gráficos de línea y los gráficos de redes de conocimiento. Finalmente, en la cuarta etapa se realizó un análisis de los resultados bibliométricos según cada laboratorio del Instituto.
Además, se escoge la base de datos Elsevier's Scopus, ya que permite la consulta y el acceso a las referencias bibliográficas en diferentes campos del conocimiento, entre ellos, la ingeniería. Según Mongeon y Hus (2016) Scopus almacena 6730 revistas en las áreas de ciencias naturales e ingeniería, lo cual corresponde al 32,9\% del total de revistas indexadas en Scopus.

\section{Resultados}

\section{Resultados de búsqueda}

Desde el año 1961 a la fecha, se presentaron los siguientes resultados de búsqueda por cada Laboratorio. Para el laboratorio de ARCOS-Lab la línea de investigación construida fue de Creación de un Sistema Versátil para Entender y Controlar Objetos, en la cual se obtuvieron 228 resultados. En CERLAB se estudió la línea de investigación sobre Control de Proceso: Validación con Equipo Experimental, la cual resultó en 351 registros. En LABCAL se definieron los Materiales de Referencia como línea de investigación y esto resultó en 459 registros. En el LIS se definió la línea de investigación de Valoración de Impacto en tiempo real, lo cual dio como resultado 751 registros. En el laboratorio de Hidráulica de identificaron 812 resultados en la línea de investigación de Infraestructura Sostenible. Finalmente, en IMARES se definió Medición de Oleaje como línea de investigación para realizar el estudio bibliométrico y se obtuvieron 1 583 resultados, y en el laboratorio Reforesta se construyó El Aprovechamiento de Recursos Renovables como línea de investigación, el cual dio como resultado: 11101 re-

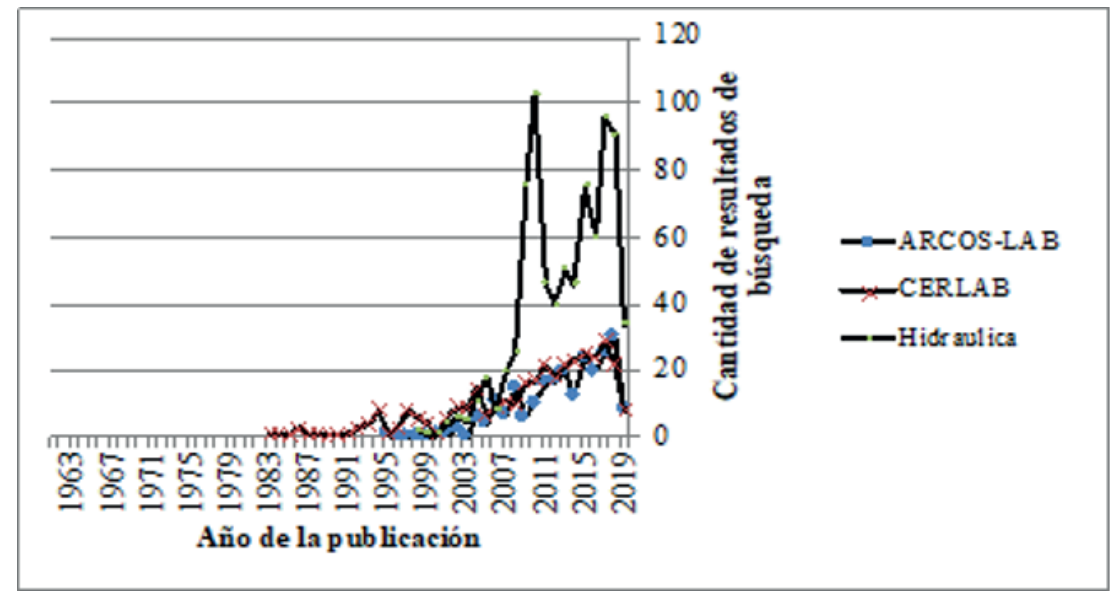

Figura 1. Comportamiento de la producción científica asociada a la línea de investigación de ARCOS-Lab, CERLab e Hidráulica. Período: 1983-2019. Fuente: elaboración propia. 
gistros. En total, los resultados de búsqueda son 15285 en las siete (7) líneas de investigación mencionadas.

\section{Comportamiento de los resultados de bús- queda}

La producción científica registrada en Elsevier Scopus, según la ecuación definida por cada laboratorio, presentó como resultado que, en todas las líneas de investigación estudiadas, el comportamiento del registro de artículos, congresos y capítulos de libros está en crecimiento. Para muestra, se evidencia este crecimiento en las figuras 1 y 2 :
La figura 1 evidencia que la producción científica en la línea de investigación de CERLab y ARCOS-Lab tiene un comportamiento de crecimiento sostenido, mientras que, en la línea propuesta por Hidráulica, el comportamiento es acelerado y bastante irregular. Dicha información fue relevante en CERLab y ARCOS-Lab para continuar invirtiendo recursos en estas líneas, ya que aparentemente en estas áreas sigue creciendo sostenidamente la producción del conocimiento a partir del año 2000, por consiguiente, es un área en consolidación, en la cual se puede aportar desde ambos laboratorios.

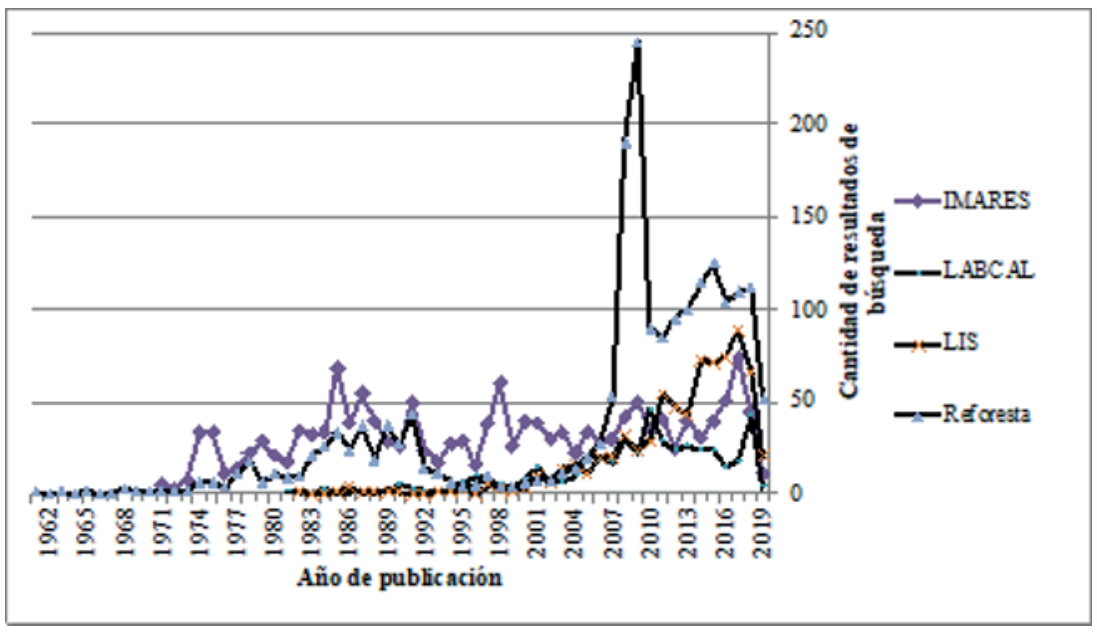

Figura 2. Comportamiento de la producción científica asociada a la línea de investigación de IMARES, LABCAL, LIS y REFORESTA. Período: 1963-2019. Fuente: elaboración propia.

La figura 2 evidencia que la producción científica en la línea de investigación de IMARES, LABCAL y el LIS tiene un comportamiento de crecimiento sostenido, mientras que, en la línea propuesta por REFORESTA, el comportamiento es acelerado e irregular. Dicha información fue tan relevante para IMARES, LABCAL y el LIS, como lo fue para CERLab y ARCOS-Lab, con el fin de orientar los esfuerzos en la escritura científica. Para REFORESTA, este resultado planteó que la ecuación de búsqueda construida aborda temas de investigación variados, por consiguiente, el comportamiento es muy variado y quebrado.

\section{Países líderes en la producción científica}

Cabe destacar que Estados Unidos de Norteamérica se encuentran en la primera posición entre países que registra mayor cantidad de resultados de búsqueda en seis de las siete líneas de investigación estudiadas, tan solo en la línea de investigación del laboratorio CERLAB, este país es desplazado a la tercera posición, y es China el primer lugar con 85 publicaciones y Japón el segundo lugar con 31. En cuanto a la posición de Costa Rica en el ranking de los diez primeros países que más publican artículos científicos en esta línea, la mejor posición es precisamente en la línea de investigación de Control de Proceso: Validación con equipo experimental, puesto que es el noveno país del mundo en cuanto la cantidad de artículos científicos, ya que reporta ocho publicaciones, de las cuales cuatro fueron publicadas por José David Rojas Fernández, investigador de CERLab.

La siguiente posición que ocupa Costa Rica es el puesto diecisiete con dos publicaciones, en la línea de investigación propuesta por el ARCOS-Lab. En las restantes cinco líneas de investigación, Costa Rica no registra publicaciones en Elsevier Scopus. 


\section{Medios de difusión de la producción cientí- fica}

Particularmente, para la línea de investigación propuesta por el ARCOS-Lab y por el Laboratorio de Hidráulica la producción científica por medio de congresos, a diferencia del resto de laboratorios del INII, es el medio de difusión más utilizado por la comunidad científica mundial. En la línea de investigación de ARCOS-Lab se han registrado 61 del total de 228 publicaciones en la materia, en tres conferencias entre las cuales destaca lo siguiente: 17th IEEE-RAS International Conference on Humanoid Robotics, Humanoids 2017, dicho hallazgo tuvo implicaciones en el plan estratégico de este laboratorio, ya que se incluyó como acción estratégica que en los años 2021, 2023 y 2025 se escribiera un artículo en alguna de las conferencias mencionadas. Por el contrario, en los restantes cinco laboratorios las revistas son el medio de difusión mayormente utilizado para divulgar la producción científica. Para CERLab destaca la revista IEEE Control Systems Magazine con 35 artículos científicos de los 351 registros recabados; para el laboratorio IMARES destaca la revista Journal of Waterway, Port, Coastal and Ocean Engineering con 168 artículos científicos de 1583 registros en Elsevier Scopus. En el LABCAL, principalmente, se publica en la revista Jounal Jiliang Xuebao/Acta Metrologica Sinica, con una cantidad de 45 artículos científicos de 459 registros. Para la línea de investigación del LIS, se obtuvieron 40 artículos científicos en la revista Bulletin of Earthquake Engineering, de 751 registros en Scopus. Finalmente, para el laboratorio REFORESTA la revista Journal Soil Biology and Biochemistry es en la que se publica mayoritariamente. Dicho hallazgo, tuvo implicaciones en los Planes estratégicos de estos laboratorios, ya que se incluyeron acciones estratégicas para dirigir la escritura de artículos en las revistas mencionadas, con excepción de REFORESTA.

\section{Cluster Map de la producción científica}

El mapa del clúster de la producción científica fue estudiado por medio de la identificación de las publicaciones que se realizaron de manera conjunta por dos o más centros de estudios ubicados en países distintos. En este sentido, cabe señalar que Costa Rica en la línea de investigación de Control de Proceso: Validación con equipo experimental del laboratorio CERLab presenta producciones científicas (los puntos amarillos) realizadas en red. Lo precedentemente destacado se visualiza en la figura 3 :

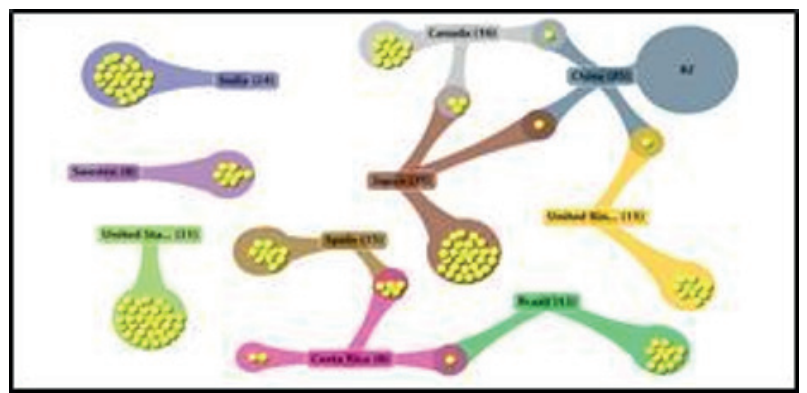

Figura 3. Cluster map de los diez primeros países que registran en colaboración publicaciones en Scopus. Línea de investigación Creación de un Sistema Versátil para entender y Controlar Objetos de CERLab. Período: 1983-2019. Fuente: elaboración propia.

\section{Conclusiones}

El analizar la producción científica mundial en áreas de conocimiento acerca del quehacer investigativo del Instituto de Investigaciones en Ingeniería (INII) de la Universidad de Costa Rica (UCR) permitió orientar estratégicamente la escritura científica acerca del Control de Proceso: Validación con equipo experimental, ya que esta muestra resultados alentadores que evidencian el protagonismo mundial del Instituto en la producción científica. Por otro lado, el análisis bibliométrico permitió identificar los medios de difusión de los resultados científicos por cada línea de investigación, en lo cual se destaca, que la comunidad científica en la mayoría de las líneas de investigación estudiadas publica en revistas científicas, mientras que en la línea de investigación: Creación de un Sistema Versátil para entender y Controlar Objetos, la comunidad científica publica artículos mayoritariamente en congresos.

La innovación tecnológica en el proceso de búsqueda de las publicaciones científicas indexadas en Elsevier Scopus es una herramienta eficaz para orientar la escritura de artículos científicos según el comportamiento de la producción científica y los resultados de búsqueda, la participación en congresos, los medios de difusión de la ciencia en cada línea de investigación, la publicación en red según los resultados del mapa clúster, lo cual tuvo implicaciones en la inclusión de acciones estratégicas dirigidas a fortalecer cada línea de investigación en el marco del Plan Estratégico 2019-2025 del INII. El estudio bibliométrico permitió orientar la escritura en cada línea de investigación, al orientarla a revistas y congresos en los cuales la comunidad científica respectiva, mayoritariamente, participa. Sin esta información y conocimiento complementa- 
rio aportado por cada equipo de investigación, el estudio bibliométrico se tornaría con poca rigurosidad científica, por consiguiente, poco veraz para orientar la escritura en el campo de la ingeniería del INII.

\section{Agradecimientos}

A Cristopher Montero Corrales y a Carmen Gómez Brenes por su revisión del artículo.

\section{Referencias}

Blanco, M. (2004). Reseña Histórica del Vigésimo Quinto Aniversario de la Fundación del Instituto de Investigaciones en Ingeniería. Revista Ingeniería. San José, Costa Rica.

Daim, T., Rueda, G., Martin, H. y Gerdsri, P. (2006). Forecasting emerging tech- nologies: Use of bibliometrics and patent analysis. TechnologicalForecastingand Social Change, 73(8), 981-1012.
López, J. (1972). El análisis estadístico y sociométrico de la literatura científica. València: Facultad de Medicina.

Mongeon, P., y Paul-Hus, A. (2016). The journal coverage of Web of Science and Scopus: a comparative analysis. Scientometrics, 106(1), 213-228.

Price, D. (1963). Little science, big science. New York: Columbia University Press.

Spinak, E. (1996). Diccionario enciclopédico de Bibliometría, Cienciometría e Infometría. Caracas: UNESCO.

Villegas, B. Rápida y pertinente búsqueda por Internet mediante operadores Booleanos." UniversitasScientiarum (2003): 51-54. 